\title{
Germany: still sleeping with the enemy?
}

W

ir sind stolz darauf, den einst von Willy Brandt beschworenen „blauen Himmel über der Ruhr" erreicht zu haben mit einem Rückgang des nationalen Staubausstoßes von 1.883 .000 Tonnen 1990 auf 209.000 Tonnen 2002. Der Grobstaub ist Historie, ein neues Staubproblem bestimmt die aktuelle Debatte - der Feinstaub. Gestützt auf fachlichen Rat, teilte der Bundesminister für Umwelt, Naturschutz und Reaktorsicherheit im November 2004 mit, dass „die Lebenserwartung aller Deutschen nach neuesten Schätzungen wegen der Feinstaubbelastung um 9 Monate“ sinke, „1-3 Monate“ gingen "auf das Konto des Diesel-Smogs."

Das „Feinstaubgespenst" geht um, es bestimmt die Schlagzeilen, seit die neuen Grenzwerte für Feinstaub (PM10) gelten: $50 \mu \mathrm{g} / \mathrm{m}^{3}$ dürfen seit 1 . Januar 2005 nur an 35 Tagen pro Jahr überschritten werden. Grundlage für diese Überlegungen waren Studien, die zeigten, dass vor allem auch kardiovaskuläre Risiken mit der Feinstaubbelastung zunehmen. Aus der Debatte wurde deutlich, dass die bereits am 27. September 1996 vom Europäischen Rat verabschiedete „Luftqualitätsrahmenrichtlinie" von den politischen Instanzen, aber auch von den betroffenen Industriezweigen nicht rechtzeitig ernst genommen worden ist. Ob die EU-Richtlinie zu ehrgeizig oder wissenschaftlich einwandfrei begründet ist, mögen andere beurteilen. So ist eines der Probleme, dass an den bundesweiten Messstellen mit PM10 eine Partikelmasse erfasst wird, die keine Rückschlüsse auf die möglicherweise gesundheitsrelevanten PM2.5 erlaubt. Ein anderes Problem ist, dass aus epidemiologischen Daten zwar Korrelation, aber keine Kausalität abgeleitet werden kann. Sicher erscheint aber, dass - anders als im be-

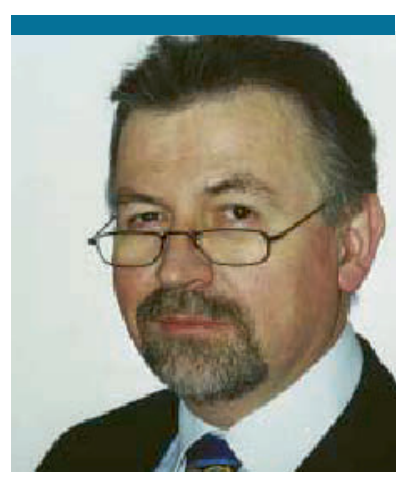

Prof. Dr. Bernhard Przybilla, Klinik und Poliklinik für Dermatologie und Allergologie, Klinikum der Ludwig-MaximiliansUniversität, München

nem mehrjährigen Verhandlungsmarathon versucht, die Regelungen des Abkommens zu entkräften, musste schließlich aber doch dem öffentlichen Druck nachgeben. Dass das Zigarettenrauchen im Vergleich zum Feinstaubproblem einen ungleich größeren Einfluss auf die Gesundheit besitzt, muss nicht umfassend begründet werden. „Der Rauch einer einzigen Zigarette produziert laut einer italienischen Studie etwa soviel Feinstaub wie ein 100 Minuten laufender Dieselmotor", meldete der Spiegel (4. April 2005). Aufgrund rein fiskalischer Überlegungen ist die Bundesregierung

nachbarten Ausland - nicht alle Möglichkeiten zur Verringerung des Feinstaubausstoßes genutzt worden sind, wie der Einsatz geeigneter Filter für Dieselkraftfahrzeuge. „Politik und Autoindustrie haben verschlafen", heißt es - oder hat die Politik Rücksicht auf die nationale Autoindustrie genommen?

Der Umgang der nati„Der Umgang der Politik mit der Feinstaubproblematik ang mit der Zigarette, an der der Staat aus wirtschaftlichen

Gründen ein hohes Interesse hat." onalen Politik mit der Feinstaubproblematik erinnert an den Umgang mit der Zigarette, an der der Staat seit langem aus wirtschaftlichen Gründen ein hohes Interesse hat. Mehr als 40 Länder haben das internationale $\mathrm{Ta}$ bakkontrollabkommen der WHO seit Inkrafttreten am 27. Februar 2005 unterzeichnet. Dieses sieht z. B.Werbeverbote, Vermeidung irreführender Bezeichnungen, wie „mild“ oder „leicht“, Maßnahmen zum Schutz vor Passivrauch und wirksame Preis- und Steuererhöhungen vor. Die Bundesregierung hat laut BÄK intern (April 2005), gemeinsam mit der US-Regierung, in ei-

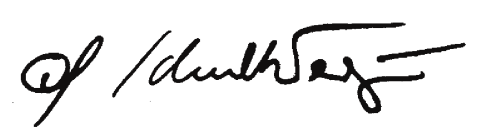

Prof. Dr. Gerhard Schultze-Werninghaus noch nicht sicher, ob die für den 1 . September 2005 geplante dritte Steuererhöhung tatsächlich umgesetzt wird - obwohl 8\% der Raucher bereits nach der ersten Steuererhöhung berichtet hatten, dass sie aus diesem Grund mit dem Rauchen aufgehört hätten (BÄK intern, April 2005).

"Germany: still sleeping with the enemy?"“ (Simpson D. Tob Control 2003;12: 343-4) - stimmt diese Formel? Die Feinstaubdebatte wie die Behandlung des $\mathrm{Zi}$ garettenrauchens belegen, dass die politische Führung bei gesundheitlichen Risiken nicht immer entschlossen die notwendigen Maßnahmen ergreift. Und dies, obwohl geklärt scheint, dass ,as with tobacco smoke and lung cancer, policymakers can't wait for all the scientific answers before taking action to prevent deaths from dirty air" (Kaiser J. Science 2005; 307: 185861). Fazit: „Der Staat schützt auch in Verantwortung für die künftigen Generationen die natürlichen Lebensgrundlagen ..." (Grundgesetz, Artikel 20 A). Man kann nur hoffen, dass er diese Aufgabe in wissenschaftlich abgesicherter und konsequenter Weise beachten möge.

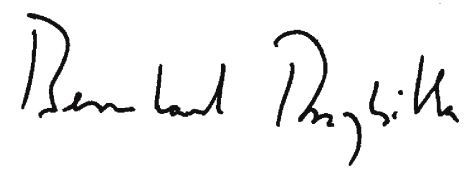

Prof. Dr. Bernhard Przybilla 